\title{
Una Interpretación Pampeana de Goethe y Gounod
}

D Emos el parabién al señor Walter Owen, de Buenos Aires, pues no sólo tradujo al inglés el Martín Fierro de José Hernández, sino que también publicó en 1943 una versión rimada en inglés del Fausto de Estanislao del Campo. Hace varios meses recibí del digno traductor una comunicación en que anunciaba la próxima entrega a las cajas de otra obra suya (por cierto muy diferente de las mencionadas) : la traducción poética de la primera parte de La Arancana de Ercilla. Cuando aparezca ésta, habremos de aplicarle el cartabón -empleando la graciosa frase de Martín Fierro- de un criterio múy distinto del que impera cuando se trata de poetas gauchescos. Pero ahora ocupémonos del Fausto y de la traducción de Owen.

Dicha versión es muy digna de elogios. Pocos contemporáneos hay que escriban sobre el gaucho con el acierto que muestra Owen. Es mi propósito indicar algo más adelante, por medio de ejemplos, algunas de las bases de este juicio, tal como se dejan ver en los versos del traductor. Por el momento, conste que el aplauso que le valió su traducción métrica del Martín Fierro en alguna crítica que hice publicar en el Boletin de Estudios Hispánicos de la Universidad de Liverpool, debe reclamarse también para su traducción del Fausto. A su pluma no le falta maestría; el tomo está bellamente ilustrado; las notas son muchas y muy sugestivas. Su introducción llama la atención por un rasgo poco común: es breve. No impide Walter Owen que lleguemos los lectores sin demora a gozar del interesantísimo relato hecho por el gaucho Anastasio, "el Pollo", a su camarada don Laguna. Consta el poema original de seis cantos: los versos de éstos alcanzan la suma de 1278 ; están agrupados en redondillas. $Y$-i sor- 
préndase quien lea estas líneas sin haber sabido nada de Del Campo! - aunque se trata nada menos que de una representación del Fausto de Gounod, vista por un gaucho en Buenos Aires, el juicio más completo y autorizado que jamás se pronunció sobre tal poema 1o emitió don Marcelino Menéndez y Pelayo y fué: "Esta es poesía buena, sana, legítima."

Siendo conocidísimas las escenas del Fausto de Gounod, baste decir que se representó por primera vez en Buenos Aires, en el Teatro Colón, el 24 de agosto de 1866. Anastasio "el Pollo" nos dice que asistía a esta función. En lenguaje perfectamente gaucho lo cuenta todo a Laguna, quien le ha encontrado inesperadamente a orillas del Plata, unos días después.

El motivo del encuentro es de los más artificiosos. En realidad, no existe tal, a menos que sea el deseo del autor hacer entrar en función a sus personajes. La historia de Goethe no es lo que más interesa a Del Campo. Este-se propone hacernos penetrar en el más hondo rincón del alma gaucha. Así se explican las largas digresiones, una de ellas en el canto tercero, que nos refiere lo que pasa en el acto segundo de la ópera - paréntesis largo que es toda una raṕsodia en honor del Plata, cuyas olas se extienden ante la vista de los dos gauchos mientras conversan; otra sobre el amor de la mujer, en el canto cuarto; también unas redondillas que pintan la llegada de la aurora; en el canto quinto, al principio, hay un trozo muy emocionante que habla del triste destino de la mujer engañada; luego una visión de crepúsculo muy romántica; por fin, en el canto sexto la comparación magistral de una niña (v. gr. Margarita) con una flor de verano.

Tales digresiones no desagradaban a los lectores de 1866, fecha de la publicación del poema; al contrario, estaban acostumbrados a escuchar a payadores que cantaban un sinfín de versos, llegando muchas veces a trasnochar, sin que los oyentes se quejasen. Si don Laguna y los lectores de Del Campo aceptaban dichos pasajes como cosa natural, era porque había en ellos una verdadera mina de sensibilidad poética; el caudaloso Plata, la vastísima pampa vista al alba o en la luz crepuscular, las flores del campo y las constelaciones del cielo argentino - todas estas manifestaciones variadas de la faz de la naturaleza eran su pan cotidiano. Pero, ¿acaso eran los pormenores de la historia de Fausto, tristes lugares comunes en la vida europea, igualmente comprensibles? 
Parece que lo eran. La guerra a la que va Valentín es para ellos la guerra del Paraguay; las diabluras de Mefistófeles en la bodega caben fácilmente en el marco de una vida extraordinariamente rica en creencias supersticiosas. En otras palabras, Del Campo halló en la acción del Fausto de Goethe un tema como pocos, apto para la confección de un "poema sano, bueno y legítimo".

¿Es buen traductor del poema de Del Campo este señor Owen, de estirpe británica? He aquí, sacado del prólogo, lo que según Owen debe hacer un traductor digno de confianza: Su deber es el de asimilar mental y emocionalmente la obra original, y entonces, si siente... que por el momento ha absorbido las características psicológicas del autor como una personalidad casi secundaria, darle rienda stelta a su propia facultad en el intento de crear de nuevo el original, esta vez en el lenguaje de él. Las formas de la versificación, los ritmos que convengan a su propósito y los límites de la libertad que se permita al traducir el texto original, deben ser fijados por su propio criterio literario. Del acierto de tal juicio depende el éxito -o el fracaso- de su trabajo.

Owen parece reconocer que se ha permitido cierta libertad; y a fe mía, la evidencia de ello no se escapa a quien lee buscándola. Precisemos. No quiero decir que ciertos "barbarismos" no convengan a una poesía de esta índole. Así se justifica, por ejemplo, "absentee" en

And calls fot a dram of absentee

Which he said was his favorite pizen.

(Pidió un ajenco y 10 trujo

E1 mozo del bodegón.-Canto III)

En cambio me parece detestable la frase "in the circs" en

They hand't much time for sweet goodbyes,

In the circs things was over brief.

(A gatas medio alcanzaron

A darse una despedida.-Canto $\mathrm{V}$ )

Incumbe a la crítica imparcial rehusar la aprobación a ciertas rimas, no porque sean jocosas sino porque se hallan grotescamente exageradas. Esto lo decimos aun admitiendo las muchas y muy se- 
rias dificultades que ha de salvar el rimador. Ejemplo de tal incongruencia son los versos siguientes:

There ain't a thing from first to last,

Will make your eyes pop as this'll-

The Devil he blows a pietcin'blast

On a thing like a watchman's whistle;

And there was a church, whacked down, kerplonck!

In the time a cock crows, or less.

-It's a metcy you weten't fotkin' a bronc!

(Todo el mundo estaba ajeno

de lo que allí iba a pasar.

cuando el Diablo hizo sonar

como un pito de sereno.

Una iglesia apareció

en menos que canta un gallo...

-iVea si dentra a caballo!

- Me larga, créamelo.-Canto V)

Recalcamos este punto, porque no parece inverosímil la conjetura de que ciertas rimas extravagantes no le disgutstan del todo al flamante versificador de Buenos Aires. Ya señalé, en el estudio mencionado arriba, algunas rimas dudosas en su traducción del Martín Fierro. Sin duda el señor Owen esgrimirá buena cantidad de argumentos para hacerlas aceptar, pero nada convencerá al que estas líneas escribe sobre que sea de buen gusto ni sentido común rimar "this'll" (hará esto) con "zhistle" (pito). Debe guardarse cierta relación de decoro entre las formas que se rimen, y vemos que tal concordancia se guarda en los versos de Del Campo. Adhiriéndose a dicha regla, hasta se permite rimar chiquero con compañero (canto IV) para indicar a éste lo despreciable (como dice Tiscornia en su nota) que parece el parque Lezama, una de las bellezas del Buenos Aires de aquel entonces, en relación con el jardín de Margarita.

Pero - falta más seria - se encuentra en cierto pasaje del canto VI una traducción que está muy lejos de ser exacta. El lector juicioso no podrá decidir si Owen sabe que caja quiere decir a veces "drum" (tambor) ; o si le dió de propósito el significado de "coffin" (ataúd) para desarrollar un concepto que le gusta. 
With never a thought, poor wandered soul, That the box they were goin' to give her, Was the one in the yatd, beside the hole, They were puttin' her in forever.

( in ver que, en su situación, la caja que la esperaba era la que redoblaba antes de la ejecución.-Canto VI)

Obsérvese cuảnto énfasis da la versión inglesa no sólo al ataúd, sino también a los detalles acompañantes del "hoyo" (hole) ya cavado en el patio de la cárcel. Júzguese pues si tal concepto vale tanto como el del tambor que redobla, en el verso de Del Campo.

Concluimos que Owen se sirve de todos los privilegios reconocidos al traductor, no solamente en lo que se refiere a la "forma" sino también al "fondo", o sea el traducir leal y puntualmente - y a veces esta frescura resulta bien, produce efectos muy pintorescos; pero en una que otra ocasión no llega a convencernos de que oímos ni la nota genuinamente gaucha, ni la absolutamente correcta. Sin embargo, tales casos no son frecuentes.

Con esta versión inglesa se completa la tríada: 1) el Fansto de Goethe-Gounod; 2) el Fausto de Del Campo; 3) el Fausto de Walter Owen. En cierto modo externo, amplio y superficial, cualquier traductor de la categoría de Owen se ve obligado a sacar inspiración no solamente de 2) sino de 1), porque el Fausto de Gounod es una ópera de situaciones universales y sobrecargadas de emoción; nadie hay más capaz que Owen de apreciarlas y verter su perfume especial en las cuartillas de su traducción. 2) es el relato, sumamente hábil, de cómo reaccionara un gaucho argentino al asistir a la representación de la obra francesa. El autor, Estanislao del Campo (1834-1880), era un porteño de abolengo distinguido, de preparación satisfactoria, bastante discreto; hombre de variadas lecturas, tuvo una intensa actuación cívica y militar. Ya había escrito varios poemas de estilo gauchesco, algo parecidos a los de Ascasubi; en ellos figuraba como protagonista el mismo "Pollo". Pero debe tenerse en cuenta que Del Campo fué urbano, culto, fino, de linaje y parentesco excepcionales. Si él salió airoso al escribir obras del género gauchesco, tal triunfo debe celebrarse con aplauso: es de veras un tour de force. $\mathrm{Y}$ su maestría estriba en el poder de penetrar en el alma 
gaucha. Caracterizan consecuentemente esta penetración muchos detalles que nacen de la vida y las costumbres gauchas; muchos trozos patéticos; otros tantos regocijados. El "traductor" de este éxito ruidoso, Walter Owen, no es ningún "traidor". Coronen su obra, pues, los laureles de un triunfo bien merecido - "una gauchada", dirían allá en su país austral- y apresurémonos a citar pruebas del mismo.

¿Se le exigen "muestras" de la vida gaucha: de cómo bebían, cabalgaban, peleaban, "reputaban el ganado"? Owen traduce así una escena de bodegón:

The Captain ups his swig of gin,

And healths the assembled gents;

As the gathetin' hoists - the Devil comes in, And ptesents his compliments.

(El capitán, con su vaso,

a los presentes brindó

$y$, en esto, se apareció

de nuevo el Diablo, amigazo. - Canto III)

Hablando de la equitación gaucha:

Its many a time he cinched his wood

On a chestnut hoss I gave him.

(Bastantes veces montó

un zaino que yo le di! - Canto II)

Así traduce el trozo que narra el duelo entre Satanás y Valentín:

But the fight was ovet when bate begun,

Old Nick gave his blade a twiddle,

And as quick as you say Jack Robinson,

The Cap's sword broke in the middle.

The Captain's eyes pop - and breathin' hard,

Yells "Satan! This fight's not level!"

Then grips the blade and shoves out the guard,

Like a crucifix at the Devil.

You ought to have seen the Devil, mate,

Bitin' steel, all a-shiver and shake... 
(No bien a tocarse van

las hojas, créameló,

la mitá al suelo cayó

del sable del capitán.

"Este es el Diablo en figura

de hombre!", el capitán gritó,

$y$, al grito, le presentó

la cruz de la empuñadura.

¿Viera al Diablo retorcerse

como culebra, aparcero!... - Canto III)

$\mathrm{Y}$, sacada de la vida ganadera que no era por cierto de las más cómodas, nos da una soberbia comparación al tratarse del río embravecido, que Del Campo compara con un campo quebrado y con un bagual, es decir, un caballo sin domar:

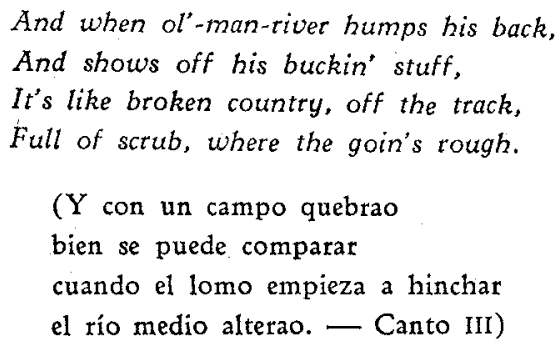

Para quien se interese en las pintorescas locuciones del gaucho, vistas a través de otro idioma, la versión de Owen ofrece muchos tesoros. Hablando de Valentín, le dice el Diablo:

- Mistet Captain, you'll sute be pronto dead;

You're tagged for the boneyard trail.

(Y le dijo: "Capitán,

pronto muere, créaló." - Canto III)

Como se ve, la frase sucinta y enérgica del traductor no pierde nada en relación con el original.

Otra comparación se lee en el trozo donde critica Laguna:

- No sea bárbaro, canejo!

¿Qué comparación tan fiera:- 
objeción que traduce Owen así:

- By hell! That's crazy comparison;

You've got your spurs crossed, I'll say!

En el último canto, la súbita huída de Satanás inspira a Owen a escribir lo siguiente:

The Devil wasn't taking the date, He was scared stiff, I'll be bound. And the same as any peludo thete, He holed-up underground.

(Pero el Diablo, que miró el sable aquel y el escudo, lo mesmito que un peludo bajo la tierra ganó. - Canto VI)

La frase "bajo la tierra ganó" o alguna de sus variantes se halla repetidas veces en la literatura gatuchesca y, como vemos, es una locución que podría desafiar a muy buenos traductores. Sin embargo, Owen halla el equivalente en inglés, aportando así nuevas riquezas en este campo literario.

Consideremos ahora el elemento sentimental en la obra de Goethe-Gounod, hallado también, por supuesto, en las redondillas de Del Campo: la sencillez de Margarita, la escena de los amantes en el jardín, la aflicción de la niña-madre y su rapto de locura en la cárcel.

Tenemos que reconocer que Owen ha reflejado estos estados sentimentales en lenguaje que parecerá a veces algo tosco, pero que generalmente es enérgico y fiel. Por extrañas que puedan considerarse algunas de las palabras, no se encuentra nada más adecuado como "muestra" de su habilidad que los versos que aquí reproducimos y que son del más puro sabor gaucho:

But a gal alone, with het world gone bust, And things closin' in on her, And her man gone off in a cloud of dustWhat's she left to stay living'for?

She'll find in hard to get toof or bed, She can weep, if her eyes aint dry, And christen with teats the cutly head Of her little one, by and by. 
Well, that was the way the doctor's gal

Went on fait piteous;

She broke clean down and cried, an'all,

Right in front of all of us.

Now, honest Injun, tell me pard,

This is what I'm gettin'at-

Wouldn't any gaucho swallow hatd

At seein' a thing like that?

-Well, I won't say it's smoke' got in my eyes;

Though I'm tryin' hatd to grin:

That's a teat-drop, sute, and double-size,

That's hangin' on my chin.

(Pero sola y despreciada

en el mundo ¿qué ha de hacer?

¿A quién la cara volver?

¿Ande llevar la pisada?

Soltar al aire su queja

será su solo consuelo,

y empapar con llanto el pelo

del hijo que usté le deja.

Pues ese dolor projundo

a la rubia la secaba,

y por eso se quejaba

delante de todo el mundo.

Aura confiese, cuñao,

que el corazón más calludo

y el gaucho más entrañudo

allí habría lagrimiao.

¿Sabe que me ha sacudido

de lo lindo el corazón?

Vea, si no, el lagrimón

que al oirlo se me ha salido....- Canto v)

Acerca de este trozo, observa el sagaz crítico Tiscornia: "El desamparo y la desgracia de Margarita, sinceramente sentidos y referidos por Anastasio, sacuden también el alma recia de Laguna." (Introducción, pág. 36, de su edición Poetas gauchescos, Buenos Aires, 1940). Así escribe Del Campo, y Owen no le va en zaga. 
Los elementos alegres de su trađucción son, naturalmente, numerosísimos, ya sea en frases sueltas:

$$
\begin{aligned}
& \text { It's Curnel Fowst, I understand, } \\
& \text { Ot my mem'ry's gettin' dim; } \\
& \text { The Uruguayan -no doctor him- } \\
& \text { Why, I served in his command! } \\
& \text { (-iDotor, dice? Coronel } \\
& \text { de la otra banda, amigazo; } \\
& \text { lo conozco a ese criollazo } \\
& \text { porque he servido con él. - Canto II), }
\end{aligned}
$$

ya sea en cuartetas como las que nos cuentan el esfuerzo del "Pollo" por arrancarle a Laguna alguna confesión de amoríos pasados:

You're in for a passel of sotrow, pard,

When love's got you roped and tied.

The wound in yout hide may get old and hatd,

But the hutt'll stay stuck inside.

-All right; I datesay it's like you tell-

I'm built different, I suppose-

But what about Fowts and the Devil? -Well,

I'll get back to that; here goes.

(Y usté, firme en su pasión...

y van los tiempos pasando,

un hondo surco dejando

en su infeliz corazón.

-Güeno, amigo: así será, pero me ha sentao el cuento...

- Qué quiere! Es un sentimiento...

Tiene razón: allá va. - Canto IV)

La lectura de esta traducción nos inclina a pensar que quizás se hayan pasado por alto en algún juicio crítico los rasgos humorísticos del gaucho, a favor del ensalzamiento de su valor y de su estoicismo, rasgos mucho más característicos en las manifestaciones literarias 'del tema. Si así fuere, habrá pocas cosas más aptas para deshacer tal creencia que la lectura de los regocijados versos de Owen.

Tratándose de una ópera cuyo original evoca tantos sentimientos de tan diversa índole como el Fausto, el crítico no debería dejar 
de observar lo bien que Owen retrata a los supersticiosos - a Laguna y al "Pollo", por ejemplo, que en cada instante prorrumpen en frases breves pero fervorosas. Citemos:

That me and the Devil... - Go easy there, Ot you'll have him here! Believe me, mate, Old Satan in petson tiz my hair The other night...

(... que el Diablo y yo... amigo! ¿no sabe usté -i Cállese, que la otra noche lo he visto al demonio?...- Canto I)

o ésta :

- Listen matey; may lightnin' strike me dead, If it ain't pute gospel truth.

(-Mire: que me caiga muerto si no es la purá verdá. - Canto II)

Por último, léase la escena del bodegón:

Before gettin' to grips the Devil made $A$ scratch between him and the other, And the floor starts blazin' behind the bladeWhat steel to light tinder, brother!

-What you sayin'! For me, give me common steel.

I don't want a fite-maker like that;

You'd be thinkin' the same, if you could feet

The stink it kicked up - my hat!

(Antes de cruzar su acero,

el Diablo el suelo rayó: ¡viera el juego que salió! ...

- Qué sable para yesquero!

- ¿Qué dice? ¡Había de oler el jedor que iba latgando mientras estaba chispiando el sabie de Lucifer! - Canto III) 
Como es de todos sabido, la escena final de la pieza de Gounod es una de las más majestuosas y emocionantes: los dos gauchos vibran intensamente al ocurrir la ascensión de Margarita de su prisión al cielo:

And there with these eyes, Laguna pal,

Don't think it's a fairy story-

I saw the soul of you little gal

Ridin' up on a cloud to glory.

(Y no crea que es historia:

yo vi entre una nubecita

la alma de la rubiecita.

que se subía a la gloria. - Canto VI)

No son grandes gemidores estos gauchos. Dice sencillamente don Laguna al "Pollo":

What I most admite is you didn't feat

To sit seein' them things you've said,

a lo que responde el "Pollo":

It's four or five days I've been feelin' queet,

Sort of seein' things in my head.

(-Lo que admiro es su firmeza

al ver esas brujerías.

- He andao cuatto o cinco días

atacao de la cabeza. - Canto VI)

Como se ve, la versión de Owen no deshonra en este detalle el original de Del Campo.

Lo antedicho encierra, pues, dos conclusiones: que el elemento nuevo que aporta Del Campo a la historia de Goethe-Gounod son los conceptos y locuciones rioplatenses, que nada quitan sino que mucho añaden al vigor e ingenuidad del relato. Owen debía traducir al inglés el original y las anotaciones - todo lo que se encuentra en el Fausto de Del Campo- en rimas que fuesen fieles al espíritu de su original. Lo ha hecho, y los contados pasajes en que hemos encontrado motivos para diferir de su técnica de traductor apenas disminuyen la excelencia de la obra. 
Ofrezcámosle otro tributo final, que lo merece: En su nota sobre el significado de la palabra gaucha, dice Owen (en las páginas 9697) : "Ningún extranjero, sobre todo nadie que tenga el temperamento anglosajón, puede llegar a simpatizar con los argentinos si no tiene presente el hecho de que ... la 'piedra de toque' de su carácter son las virtudes gauchas de generosidad, hospitalidad, devoción a sus amigos, confianza en sí mismos, valor personal y amor intenso a la libertad física. De ahí viene que, según nuestro criterio, sean rebeldes a toda sujeción y censura ajenas, vehementes, no muy aptos para el 'team-work', o sea el trabajo en conjunto con los demás miembros de un equipo o compañía cualquiera, quisquillosos con respecto al amor propio o nacional y poco dispuestos a olvidar desaires verdaderos o imaginarios. Una cantidad limitada de tales caracteristicas es inherente a todo pueblo joven y vigoroso, pero, a mi modo de ver, se les ha dado mucho énfasis en el carácter nacional argentino, probablemente como consecuencia de ciertos factores que han contribuido a su desarrollo. Estos son: lo que se heredó psicológicamente de los gauchos, víctimas de intensa explotación social; el hecho de que la Intendencia de Buenos Aires ... estuvo subordinada política, económica y culturalmente al virreinato del Perú; la crecida dependencia de la Argentina del capital extranjero y de los brazos de los inmigrantes y la situación geográfica de la República, que se hallaba tan distante de los centros de donde recibía su cultura y su sostenimiento económico. El efecto de tales elementos deprimentes durante la formación de la nación argentina no pudo haber sido vencido a menos que se hubiera inferido daño a su afirmación de sí misma, que se mantuvo (y se mántiene) porfiada, si bien es verdad que dicha afirmación parece ahora haber sobrevivido a su utilidad. La mejor comprensión de esta idiosincrasia nacional traería ventajas tanto a argentinos como a extranjeros..."

Quien tal escribió, comprende; y mejor aún, ama...

Henry A. Holmes, The College of the City of New York. 
